\title{
Contraceptive utilization and associated factors among women in the extended post-partum period in wukro town, Tigray, Ethiopia: a community based cross sectional study
}

Natnael Etsay Assefa ( $\square$ natty416@gmail.com )

Adigrat University College of Health Sciences

Yodit Zewdie Berhe

Mekelle University

Weldu Mammo Werid

Adigrat University College of Health Sciences

Meresa Berewo Mengesha

Adigrat University College of Health Sciences

Kidanemariam Berhe Tekola

Adigrat University College of Health Sciences

Gdiom Gebreheat Abady

Adigrat University College of Health Sciences

Tesfay Tsegay Gebru

Adigrat University College of Health Sciences

Lidya Zewdie Berhe

Bahir Dar University

Mulu Gebretsadik Weldemariam

Adigrat University College of Health Sciences

Research article

Keywords: Contraceptive, utilization, postpartum, wukro town, cross sectional

Posted Date: November 2nd, 2019

DOI: https://doi.org/10.21203/rs.2.12957/v2

License: (c) (1) This work is licensed under a Creative Commons Attribution 4.0 International License.

Read Full License 
The authors have withdrawn this preprint from Research Square 